\title{
Examining the Function of Meromorphic with Using the Linear Convolution Operator
}

\section{Hasan ŞAHIN ${ }^{1 *}$, İsmet YILDIZ ${ }^{2}$}

\begin{abstract}
In this study, it is mentioned that meromorphic functions are univalent functions that are analytical everywhere. Complex analytical transformations were investigated by mentioning the necessary form for $f(z)$ to have meromorphic function. It is a function that satisfies the condition $h(z) \neq 0$. For analytic functions of $f$ and $g$ in the D unit disk, $f(z)$ shows the meromorphic function class with $P$ and subclasses of the $P$ meromorphic analytical function class using the subordination principle between functions with the help of Hadamard product and linear operators. In this way proves is provided.
\end{abstract}

Keywords: Meromorphic function, Univalent function, Hadamard product.

${ }^{1}$ Hasan ŞAHIN (Orcid ID: 0000-0002-5227-5300), Düzce University, Faculty of Arts and Sciences, Department of Mathematics, Düzce, Turkey

${ }^{2}$ İsmet YILDIZ (Orcid ID: 0000-0001-7544-4835), Düzce University, Faculty of Arts and Sciences, Department of Mathematics, Düzce, Turkey

* Corresponding Author: Hasan ŞAHIN e-mail: hasansahin13@gmail.com 


\section{INTRODUCTION}

The univalent function was first introduced by Alexander (1915). Nevanlinna (1921) also made detailed research on this subject. Later, univalent functions were investigated by Marx (1932), Strohhacker (1933), McGregor (1975), and many more researchers. About meromorphic functions in unit circle; Pommerenke (1963), Miller (1970), Nunokawa and Ahuja (2001) and many other researchers. Linear transformations of meromorphic functions in the unit circle were first given by Royster (1963). Later, Liu and Owa (1998) and many researchers worked on this issue.

In the middle of the 19th century studies conducted by Weierstrass, Mittag-Leffler and Picard were the beginning of systematic studies of the theory of meromorphic functions. Weierstrass, MittagLeffler theorems provide a general description of the structures of complete and meromorphic functions. The representation of the complete functions created by Weierstrass with an infinite product is the basis for the studies on the properties of these functions (Gonchar et al., 2001) .

A meromorphic function is a univalent and analytical function, but it should probably go to infinity like a polynomial in a discrete subset of this function definition set and in these singularities (that is, these exceptional points should be polar, not necessary singularity). If we define a simple finding for which a meromorphic function $f(z)$ is

$$
f(z)=\frac{g(z)}{h(z)}
$$

Here, the functions $g(z)$ and $h(z)$ are named entire function that satisfy the condition $h(z) \neq 0$ (Krantz, 1999). A meromorphic function is only finite order, isolated poles and zeros, and it does not have the necessary singularities in its definition domain. A meromorphic function where the number of poles is infinite is expressed on the drilled disk $D^{*}=\{z: 0<|z|<1\}$. For example, a Gamma function in the entire complex plane is meromorphic. It is defined as the equivalent of meromorphic function as a complex analytical transformation in the Riemann sphere. In the whole complex planet he Gamma function is meromorphic (Pandey, 2008).

Let the $h(z)$ analytic functions with $h(0)=1$ be class $A . D=D^{*} \cup\{0\}$ open unit disk is analytical. Hence;

$$
\operatorname{Re} h(z)>0(z \in D) .
$$

We can say that $f(z)$ and $g(z)$ analytically in the unit disk $D, f$ is subordined to $g$ and written as $f \prec g$;

$$
f(z) \prec g(z) \quad(z \in D) .
$$

If $\mathrm{D}$ has an analytical function of $v(z)$, it is $v(z) \leq|z|$ and $f(z)=g(v(z))$. Also, if the $g$ function is univalent in unit disk $\mathrm{D}$ then

$$
f(z) \prec g(z) \Leftrightarrow \quad f(0)=g(0) \text { and } f(D)=g(D) \quad(z \in D) .
$$

Let the $h(z)$ analytic functions with $h(0)=1$ be class $A . D=D^{*} \cup\{0\}$ analytic on the open unit disk and where

$$
\operatorname{Re} h(z)>0\left(z \in D^{*}\right) .
$$

If $\mathrm{D}$ has an analytical function, then we can write $v(z) \leq|z|$ and $f(z)=g(v(z)) \quad\left(z \in D^{*}\right)$. Also, if function $g(z)$ is univalent in $D$ then

$$
f(z) \prec g(z) \Leftrightarrow f(0)=g(0) \text { and } f(D) \subseteq g(D)\left(z \in D^{*}\right) .
$$




\section{MATERIALS AND METHODS}

Let show normalized meromorphic function $f(z)$ with class $\mathrm{P}$ (Cho and Owa, 2004).

$$
f(z)=\frac{1}{z}+\sum_{n=1}^{\infty} a_{n} z^{n}
$$

This function $f(z)$ is univalent and analytical on the drilled unit disc $D^{*}$. For function $f_{i}(z),(\mathrm{i}=1 ; 2)$ defined by

$$
f_{i}(z)=\frac{1}{z}+\sum_{n=1}^{\infty} a_{n, i} z^{n}
$$

functions $f_{1}(z)$ and $f_{2}(z)$ for the Hadamard product

$$
\left(f_{1} * f_{2}\right)(z)=\frac{1}{z}+\sum_{n=1}^{\infty} a_{n, 1} a_{n, 2} z^{n} \quad(\text { Mogra, 1991). }
$$

Let's define the function $\partial(\alpha, \beta ; z)$ by

$$
\partial(\alpha, \beta ; z)=\frac{1}{z}+\sum_{n=0}^{\infty}\left|\frac{(\alpha)_{n+1}}{(\beta)_{n+1}}\right| z^{n}
$$

for $\beta \neq 0,-1,-2, \ldots$ and $\alpha \in \square /\{0\}$ we use that Pochhammer symbol $(\lambda)_{n}=\lambda(\lambda+1)_{n+1}$. From here $(\alpha)_{n+1}=\alpha(\alpha+1)(\alpha+2) \ldots(\alpha+n)$ and $(\beta)_{n+1}=\beta(\beta+1) \ldots(\beta+n)$ if we put $(\alpha)_{n+1}$ and $(\beta)_{n+1}$ in place for

$$
\partial(\alpha, \beta ; z)=\frac{1}{z}+\sum_{n=0}^{\infty}\left|\frac{\alpha(\alpha+1)(\alpha+2) \ldots(\alpha+n)}{\beta(\beta+1) \ldots(\beta+n)}\right| z^{n}
$$

If we multiply the equation by $(1)_{n}$ and divide it by $n$ !, We find it as

$$
\partial(\alpha, \beta ; z)=\frac{1}{z} \sum_{n=0}^{\infty} \frac{(1)_{n}(\alpha)_{n}}{(\beta)_{n}} \frac{z^{n}}{n !}
$$

where

$$
{ }_{2} F_{1}(b, \alpha, \beta ; z)=\sum_{n=0}^{\infty} \frac{(b)_{n}(\alpha)_{n}}{(\beta)_{n}} \frac{z^{n}}{n !}
$$

is a known gauss hypergeometric function. If we use a familiar gauss hypergeometric function, we get

$$
\partial(\alpha, \beta ; z)=\frac{1}{z}{ }_{2} F_{1}(1, \alpha, \beta ; z) .
$$

Let define $K^{*}(\alpha, \beta)$, a new linear operator on $P$ using the Hadamard product for the function $f(z) \in P$ corresponding to $\partial(\alpha, \beta ; z)$.

$$
\begin{gathered}
K^{*}(\alpha, \beta) f(z)=\partial(\alpha, \beta ; z) * f(z) \\
=\frac{1}{z}+\sum_{n=1}^{\infty}\left|\frac{(\alpha)_{n+1}}{(\beta)_{n+1}}\right| a_{n} z^{n} \quad(1)
\end{gathered}
$$

Meromorphic functions with generalized hypergeometric functions have been discussed recently( Cho and Kim , 2007), (Dziok and Srivastava, 2003).

Fort he function $f \in K^{*}(\alpha, \beta) f(z)$ we describe $I^{0}(K(\alpha, \beta) f(z))=K(\alpha, \beta) f(z)$ and for $\mathrm{b}=1,2,3, \ldots$ 


$$
\begin{gathered}
\left.I^{b}(K(\alpha, \beta) f(z))=z\left(I^{b-1} K(\alpha, \beta) f(z)\right)\right)^{\prime}+\frac{2}{z} \\
=\frac{1}{z}+\sum_{n=1}^{\infty} n^{k}\left|\frac{(\alpha)_{n+1}}{(\beta)_{n+1}}\right| a_{n} z^{n}
\end{gathered}
$$

It follows from the equation in (1) and (Srivasta and Owa, 1986) that

$$
z(K(\alpha, \beta) f(z))^{\prime}=\alpha K(\alpha+1, \beta) f(z)-(\alpha+1) K(\alpha, \beta) f(z) .
$$

In addition, (2) above;

$$
z\left(I^{b} K(\alpha, \beta) f(z)\right)^{\prime}=\alpha I^{b} K(\alpha+1, \beta) f(z)-(\alpha+1) I^{b} K(\alpha, \beta) f(z)
$$

is arranged in the form. During this study, we assume

$$
a \in \sqcap, \beta \notin \Pi_{0}^{-}, \quad \delta_{a}^{i}=\exp \left(\frac{2 \pi i}{a}\right)
$$

and

$$
f_{a}(\alpha, \beta ; z)=\frac{1}{a} \sum_{i=0}^{a-1} \delta_{a}^{i}(K(\alpha, \beta) f)\left(\delta_{a}^{i} z\right) \quad f \in P
$$

Also we find

$$
\begin{array}{r}
f_{a, b}(\alpha, \beta ; z)=I^{b} f_{a}(\alpha, \beta ; z) \\
=\frac{1}{a} \sum_{i=0}^{a-1} \delta_{a}^{i(b+1)}\left(I^{b} K(\alpha, \beta) f\right)\left(\delta_{a}^{i} z\right), \mathrm{b}=1,2,3, \ldots
\end{array}
$$

Obviously we can write $f_{1}(\alpha, \beta ; z)=K(\alpha, \beta) f(z)$ for $b=0$ and $a=1$. Using the principle of subordination between the $K(\alpha, \beta)$ linear operator and analytical functions, we will create and investigate the following subclasses of the meromorphic analytical function class $P$ :

$$
P_{a, b}(\alpha, \beta ; h), N_{a, b}(\alpha, \beta ; h) \text { with } h \in A .
$$

Definition 1: The determination that $f \in P$ is a function from the class $P_{a, b}(\alpha, \beta ; h)$ is available when it satisfies the following subordination condition:

$$
\frac{-z\left(I^{b} K(\alpha, \beta) f\right)^{\prime}(z)}{f_{a, b}(\alpha, \beta ; z)} \prec h(z), z \in D, h \in A \text { ve } f_{a, b}(\alpha, \beta ; z) \neq 0 .\left(z \in D^{*}\right) .
$$

Definition 2: The determination that a function $f \in P$ is from the class $N_{a, b}(\alpha, \beta ; h)$ is available when it satisfies the following subordination condition:

$$
\frac{-z\left(I^{b} K(\alpha, \beta) f\right)^{\prime}(z)}{g_{a, b}(\alpha, \beta ; z)} \prec h(z), \quad(z \in D)
$$

$g \in P_{a, b}(\alpha, \beta ; h), h \in A$ and $g_{a, b}(\alpha, \beta ; z) \neq 0$ is defined as in (4).

Definition 3: The determination that a function $f \in P$ is from the class $N_{a, b}(v, \alpha, \beta ; h)$ is available when it satisfies the following subordination condition:

$$
-v \frac{z\left(I^{b} K(\alpha+1, \beta) f\right)^{\prime}(z)}{g_{a, b}(\alpha+1, \beta ; z)}-(1-v) \frac{z\left(I^{b} K(\alpha, \beta) f\right)^{\prime}(z)}{g_{a, b}(\alpha, \beta ; z)} \prec h(z), \quad z \in D
$$

for some $v(v \geq 0)$ and $g \in P_{a, b}(\alpha, \beta ; h)$, where $h \in A$ and $g_{a, b}(\alpha+1, \beta ; z) \neq 0$. To prove the main results are following our need for some trades. 
Lemma 1: Let $a(a \geq 0)$ and $\gamma$ be complex numbers. Let $\mathrm{h}(\mathrm{z})$ be analytical and convex univalent in unit disk $D$

$$
\operatorname{Re}\{\operatorname{ah}(z)+\gamma\}>0 .
$$

If $s(z)$ is analytical within $\mathrm{D}$ with $\mathrm{s}(0)=\mathrm{h}(0)$ then subordination

$$
s(z)+\frac{z s^{\prime}(z)}{a s(z)+\gamma} \prec h(z),(z \in D)
$$

implies that $s(z) \prec h(z) \quad(z \in D)$ (Miller and Mocanu, 1985).

Lemma 2: Let the function $\mathrm{h}(\mathrm{z})$ in the unit disk $\mathrm{D}$ be convex univalent and analytical. Also v(z) get analytically in unit disk D

$$
\operatorname{Re} v(z) \geq 0 \quad(z \in D) .
$$

If $s(z)$ is analytical and $s(0)=h(0)$ at $D$, then subordination $s(z)+v(z) z s^{\prime}(z) \prec h(z)$, implies $(z \in U)$ that $s(z) \prec h(z)$.

Lemma 3: Let $f \in P_{a, b}(\alpha, \beta ; h)$. In that case

$$
-\frac{z\left(f^{\prime}{ }_{a, b}(\alpha, \beta ; z)\right)}{f_{a, b}(\alpha, \beta ; z)} \prec h(z) \quad(z \in D) .
$$

Proof: Using the equation in (4),

$$
\begin{gathered}
f_{a, b}\left(\alpha, \beta ; \delta_{a}^{i} z\right)=\frac{1}{a} \sum_{c=0}^{a-1} \delta_{a}^{c(b+1)}\left(I^{b} K(\alpha, \beta) f\right)\left(\delta_{a}^{c+i} z\right) \\
=\frac{\delta_{a}^{-i}}{a} \sum_{c=0}^{a-1} \delta_{a}^{c(b+1)+i}\left(I^{b} K(\alpha, \beta) f\right)\left(\delta_{a}^{c+i} z\right)=\delta_{a}^{-i} f_{a, b}(\alpha, \beta ; z),
\end{gathered}
$$

$(i \in\{0,1, \ldots, m-1\})$ and

$$
f_{a, b}^{\prime}(\alpha, \beta ; z)=\frac{1}{a} \sum_{c=0}^{a-1} \delta_{a}^{i(b+2)}\left(I^{b} K(\alpha, \beta) f\right)^{\prime}\left(\delta_{a}^{i} z\right)
$$

Therefore

$$
\begin{gathered}
-\frac{z\left(f_{a, b}^{\prime}(\alpha, \beta ; z)\right)}{f_{a, b}(\alpha, \beta ; z)}=-\frac{1}{a} \sum_{i=0}^{a-1} \frac{\delta_{a}^{i(b+2)} z\left(I^{b} K(\alpha, \beta) f\right)^{\prime}\left(\delta_{a}^{i} z\right)}{f_{a, b}(\alpha, \beta ; z)} \\
=-\frac{1}{a} \sum_{i=0}^{a-1} \frac{\delta_{a}^{i b} z\left(I^{b} K(\alpha, \beta) f\right)^{\prime}\left(\delta_{a}^{i} z\right)}{f_{a, b}\left(\alpha, \beta ; \delta_{a}^{i} z\right)} \quad(z \in D)
\end{gathered}
$$

Since $f \in P_{a, b}(\alpha, \beta ; h)$ following that

$$
-\frac{\delta_{a}^{i} z\left(I^{b} K(\alpha, \beta) f\right)^{\prime}\left(\delta_{a}^{i} z\right)}{f_{a, b}\left(\alpha, \beta ; \delta_{a}^{i} z\right)} \prec h(z)
$$

$(z \in D, \quad i \in\{0,1, \ldots, b-1\})$ is obtained. Since $h(z)$ is convex univalent in $D$, we conclude that the result of the equation from (6) and (7), as a result, (5) is correct. 


\section{RESULTS AND DISCUSSION}

Teorem 1 : Let $h \in A$ and

$$
\operatorname{Re} h(z)<1+a \quad(z \in D, a>0) .
$$

If $f \in P_{a, b}(\alpha+1, \beta ; h)$ then $f \in P_{a, b}(\alpha, \beta ; h)$ and $f_{a, b}(\alpha, \beta ; z) \neq 0$ with the condition $\left(z \in D^{*}\right)$.

Proof: From (3) and (4),

$$
\begin{gathered}
(\alpha+1) f_{a, b}(\alpha, \beta ; z)+z f_{a, b}^{\prime}(\alpha, \beta ; z)=\frac{\alpha}{a} \sum_{i=0}^{a-1} \delta_{a}^{i(b+1)}\left(I^{b} K(\alpha+1, \beta) f\right)^{\prime}\left(\delta_{a}^{i} z\right) \\
=\alpha f_{a, b}(\alpha+1, \beta ; z), \quad(f \in P) .
\end{gathered}
$$

$f \in P_{a, b}(\alpha+1, \beta ; h)$ and let's suppose that

$$
v(z)=-\frac{z\left(f_{a, b}^{\prime}(\alpha, \beta ; z)\right)}{f_{a, b}(\alpha, \beta ; z)} .
$$

Then $v(z)$ is analytical within unit $\operatorname{disk} D, v(0)=1$, from (9) and (10)

$$
\alpha+1-v(z)=\alpha \frac{f_{a, b}(\alpha+1, \beta ; z)}{f_{a, b}(\alpha, \beta ; z)} .
$$

Here if we take the logarithmic derivative of both sides of (11) with respect to $\mathrm{z}$ and use (10), we obtain

$$
v(z)+\frac{z v^{\prime}(z)}{\alpha+1-v(z)}=\frac{z\left(f_{a, b}^{\prime}(\alpha+1, \beta ; z)\right)}{f_{a, b}(\alpha, \beta ; z)}
$$

(12) and from Lemma 3 (if replaced $\alpha+1$ )

$$
v(z)+\frac{z v^{\prime}(z)}{\alpha+1-v(z)} \prec h \quad(z \in D)
$$

From (8) and (13) with the application of lemma 4 now we get

$$
s(z)=\frac{-z\left(I^{b} K(\alpha, \beta) f\right)^{\prime}(z)}{f_{a, b}(\alpha, \beta ; z)}
$$

If we create it as (14), $s(z)$ provides the analytical $s(0)=1$ condition in $D$.

From (3) and (14)

$$
f_{a, b}(\alpha, \beta ; z) s(z)=-\alpha I^{b} K(\alpha+1, \beta) f(z)+(1+\alpha) I^{b} K(\alpha, \beta) f(z) .
$$

By differentiating both sides, according to $\mathrm{z}$ and using (14),

$$
z s^{\prime}(z)+\left(\alpha+1+\frac{z\left(f_{a, b}^{\prime}(\alpha, \beta ; z)\right)}{f_{a, b}(\alpha, \beta ; z)}\right) s(z)=-\frac{\alpha z\left(I^{b} K(\alpha+1, \beta) f\right)^{\prime}(z)}{f_{a, b}(\alpha, \beta ; z)} .
$$

Because of this (9), (10) and (15) equations we obtain the equation

$$
s(z)+\frac{z s^{\prime}(z)}{\alpha+1-v(z)}=\frac{z\left(I^{b} K(\alpha+1, \beta) f\right)^{\prime}(z)}{f_{a, b}(\alpha+1, \beta ; z)} \prec h(z) \quad(\mathrm{z} \in \mathrm{D}),
$$

and $f \in P_{a, b}(\alpha+1, \beta ; h) . \operatorname{Re}\{\alpha+1-v(z)\}>0$ is obtained. Thus (16) and Lemma 2 we get the results from 


$$
s(z) \prec h(z) \quad(z \in D),
$$

This equation implies that it is $f \in P_{a, b}(\alpha, \beta ; h)$ and thus the proof of Teorem 1 is completed.

Teorem 2: Let $h \in A$ and

$$
\operatorname{Re} h(z)<1+\alpha \quad(z \in D, \quad \alpha>0) .
$$

If $g \in P_{a, b}(\alpha+1, \beta ; h)<1+\alpha$ according to $f \in N_{a, b}(\alpha+1, \beta ; h)$,

then $f \in N_{a, b}(\alpha, \beta ; h)$ on condition that $g_{a, b}(\alpha, \beta ; z) \neq 0 \quad\left(z \in D^{*}\right)$.

Proof: According to conjecture of Theorem 2, $g \in P_{a, b}(\alpha+1, \beta ; h)$ and we obtained

$$
\frac{-z\left(I^{b} K(\alpha+1, \beta) f\right)^{\prime}(z)}{g_{a, b}(\alpha+1, \beta ; z)} \prec h(z) \quad(\mathrm{z} \in \mathrm{D})
$$

In addition, from Theorem $1 g \in P_{a, b}(\alpha, \beta ; h)$ and Lemma 3

$$
\theta(z)=-\frac{z g_{a, b}^{\prime}(\alpha, \beta ; z)}{g_{a, b}(\alpha, \beta ; z)} \prec h(z) \quad(z \in D) .
$$

Let's assume that

$$
s(z)=-\frac{z\left(I^{b} K(\alpha, \beta) f\right)^{\prime}(z)}{g_{a, b}(\alpha, \beta ; z)} .
$$

Using (3), $g_{a, b}(\alpha, \beta ; z)$ can be written as follows.

$$
g_{a, b}(\alpha, \beta ; z) s(z)=-\alpha I^{b} K(\alpha+1, \beta) f(z)+(1+\alpha) I^{b} K(\alpha, \beta) f(z) .
$$

Let's take the derivative of both sides of the equation (19) according to z, and using (9) (replaced by f, g) we find the equation

$$
s(z)+\frac{z s^{\prime}(z)}{\alpha+1-\theta(z)}=-\frac{z\left(I^{b} K(\alpha+1, \beta) f\right)^{\prime}(z)}{g_{a, b}(\alpha+1, \beta ; z)} \quad(z \in D)
$$

Using (17) and (20) together, we get the equation

$$
s(z)+\frac{z s^{\prime}(z)}{\alpha+1-\theta(z)} \prec h(z) \quad(z \in D)
$$

As a result, considering (17), (18) and (21) from Lemma 2

$$
s(z) \prec h(z) \quad(z \in D)
$$

is written. This shows that $g \in P_{a, b}(\alpha, \beta ; h)$ according to $f \in N_{a, b}(\alpha, \beta ; h)$. In this way, our proof is completed.

\section{CONCLUSION}

In this article, $P_{a, b}(\alpha, \beta ; h)$ and $N_{a, b}(\alpha, \beta ; h)$, a new subclass formed by a $\mathrm{P}$ class, are presented. It is given how $P_{a, b}(\alpha, \beta ; h)$ and $N_{a, b}(\alpha, \beta ; h)$ subclasses are created. At the same time, the subordination method and the Hadamard product were used. It was investigated by mentioning the necessary form of complex analytic transformations to have meromorphic function. 


\section{REFERENCES}

Alexander JW, 1915. Functions which map the interior of the unit circle upon simple regions, Annals of Math. 17, 15-22.

Cho NE, Owa S, 2004. Partial Sums of certain meromorphic functions, J. Inequal. Pure and Appl. Math., 5(2) Art. 30.

Cho NE, Kim IH, 2007. Inclusion properties of certain classes of meromorphic functions associated with the generalized hypergeometric function, Appl. Math. Compu., 187 (1), 115121.

Dziok J, Srivastava HM, 2003. Certain subclasses of analytic functions associated with the generalized hypergeometric function, Integral Transforms Spec. Funct., 14 (1) ,7-18.

Gonchar AA, Havin VP, Nikolski NK, 2001. Complex Analysis I. Springer-Verlag, pp 4, Newyork.Goodman A W, 1983. Univalent functions, Vol. I, Mariner Publ. Co., Tampa, FI..

Krantz SG, 1999. Handbook of Complex Variables, , Birkhuser, Boston, MA.

Liu J, Owa S, 1998. On certain meromorphic p-valent functions, Taiwanese J. Math. 2(1) , pp. 107-110.

MacGregor TH, Thomas HA, 1974/75. Subordination for convex functions of order $\alpha$. J. London Math. Soc. (2) 9, 530-536.

Marx A, 1936. Untersuchungen, ber schlichte Funktionen, Math. Ann. 107, 40-67.

Miller SS, Mocanu PT, 1985. On some classes of first order differential subordinations, Michigan Math. J. , $32,185-195$.

Miller J, 1970. Convex meromorphic mappings and related functions, Proc. Amer. Math. Soc., 25 , 220 228.

Mogra L, 1991. Hadamard product of certain meromorphic univalent functions, J. Math. Anal. Appl., 157 ,10-16.

Nevanlinna R, 1921. über die konforme Abbildung von Sterngebieten, Ofvers. Finska 58 Vet. Soc. Förh., 53 (A), Nr. 6.

Nunokawa M, Ahuja OP, 2001. On meromorphic starlike and convex functions, Indian Pure Appl. Math., 32(7), 1027-1032.

Pommerenke Ch, 1963. On meromorphic starlike functions. Pacific J. Math. 13 221-235.

Royster WC, 1963. Meromorphic Starlike, Trans. Amer. Math. Soc. Vol. 107, No. 2 , pp. 300-308.

Srivastava HM, Owa S, 1986. A certain one parameter additive family of operators defined on analytic functions, Journal of mathematical analysis and applications 118, 80-87.

Strohhacker E, 1933. Beitrage zur Theorie der schlichten Funktionen, Math. Z. 37, 356-380. 